\section{Latente Tuberkulose bei HIV: Einheitliche Screening-Strategien notwendig}

White $\mathrm{HA}$ et al. Latent tuberculosis infection screening and treatment in HIV: insights from evaluation of UK practice. Thorax 2016; DOI 10.1136/thoraxjnl-2016-209063

HIV-Patienten haben ein erhöhtes Risiko, durch Reaktivierung einer latenten Tuberkulose (LTB) eine aktive Infektion zu entwickeln. In Großbritannien wird daher von der British HIV Association (BHIVA) und dem National Institute for Health and Care Excellence (NICE) bei diesen Patienten ein Screening auf eine LTB empfohlen, wenngleich mit unterschiedlichen Strategien. Wenig ist allerdings darüber bekannt, wie diese Empfehlungen umgesetzt werden.

Um diese Frage zu beantworten, entwickelten die Autoren einen Online-Fragebogen, zu dessen Evaluation sie jeweils einen Experten jedes HIV-Gesundheitsdienstleisters in Großbritannien einluden. Es wurden insgesamt 188 geografische Regionen im Land identifiziert, in denen Daten zur HIV-Prävalenz und zur Inzidenz einer latenten Tuberkulose vorlagen.

Antworten erhielten die Autoren von 116 Personen aus 162 unterschiedlichen geografischen Regionen, da manche von ihnen mehrere Regionen betreuten. Die Antwortrate lag insgesamt bei $86 \%$ (162/188). Zwischen Regionen, die antworteten und solchen, die nicht antworteten, gab es keinen Unterschied bezüglich der Raten an HIV und Tuberkulose. Nur 57,4\% der Regionen (93/162) gaben an, ein Screening auf LTB in irgendeiner Form anzubieten; auch hier zeigte sich kein Unterschied bezüglich der Belastung mit HIV und Tuberkulose. In Regionen, die ein Screening anboten, nutzten 61,3\% (57/93) die Zahl an CD4-Zellen als Kriterium dafür, wobei 53 von 57 (93\%) eine

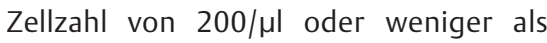
Grenzwert nahmen, die anderen benutzten höhere Zellzahlen. 80,6\% (75/93) entschieden sich anhand des Herkunfts- lands der Patienten für oder gegen ein Screening, wobei in allen Regionen Patienten aus Ländern mit hoher Tuberkulose-Inzidenz gescreent wurden. Weniger als zwei Drittel der Regionen screenten jedoch bei Patienten mit einer anderen Herkunft. Von den verschiedenen Tests wurde am häufigsten ein Essay zur Freisetzung von Interferon Gamma genutzt; 47,3\% (44/93) bzw. 45,2\% (42/ 93) verwendeten den QuantiFERONbzw. T.SPOT-Test, andere Testverfahren wurden seltener eingesetzt. Komplett an die Leitlinien von BHIVA und NICE hielten sich lediglich 35,5\% (33/93) bzw. 6,5\% (6/93) der geografischen Regionen. Von den Regionen, die kein Screening anboten, glaubten $45 \%$ (31/69), dass ihre Bevölkerung ein niedriges Risiko für eine LTB aufwies, 29\% (20/69) vertrauten den Leitlinien nicht und 17,4\% (12/69) waren der Ansicht, dass die Tests zu teuer seien.

\section{FAZIT}

Nach Ansicht der Autoren besteht ein dringender Bedarf prospektiv zu evaluieren, welchen HIV-Patienten man ein Screening auf eine latente Tuberkulose anbieten soll, welches die optimale Screening-Strategie ist und wie die Kosteneffektivität eines solchen Screenings aussieht. Eine einheitliche nationale Leitlinie sei daher erforderlich.

Dr. med. Johannes Weiß, Bad Kissingen 